\title{
EFFECT OF TRAMADOL ON POST OPERATIVE ANALGESIA WHEN ADDED TO LIGNOCAINE IN INTRA VENOUS REGIONAL ANESTHESIA (BIER'S BLOCK) FOR UPPER ARM ORTHOPEDIC SURGERIES.
}

\author{
Dr. Vivek Chakole, Dr. Ritesh Dixit, Dr. Gayatri Tadwalkar
}

1. Assistant Professor, Department of Anaesthesiology, R. D. Gardi Medical College, Ujjain (MP).

2. Assistant Professor, Department of Anaesthesiology, R. D. Gardi Medical College, Ujjain (MP).

3. Associate Professor, Department of Anaesthesiology, Government Medical College, Aurangabad (MS).

\section{CORRESPONDING AUTHOR}

Dr. Vivek Chakole. Assistant Professor,

Department of Anaesthesiology,

R. D. Gardi Medical College, Ujjain, MP 456006,

E-mail: drvivekchakole@rediffmail.com,

Ph: 00919630024978,00917368261280

\begin{abstract}
BACKGROUND: Intravenous regional anesthesia (IVRA) is known for its simplicity, effectiveness, safety, reliability and being economical for day care and emergency surgery circumventing the problems of full stomach. OBJECTIVE: Comparison of effect of lignocaine alone and lignocaine with Tramadol in surgery of upper extremity under intravenous regional anaesthesia. MATERIAL AND METHOD: In our randomized prospective study, total 60 adult ASA class I and II patients undergoing upper limb surgeries were given IVRA and studied for addition of Tramadol on intra operative pain and post operative analgesia. Patients received $0.5 \%$ lignocaine $40 \mathrm{ml}$ in one group and we added Tramadol $100 \mathrm{mg}$ in the other group. All the patients were monitored for onset of effect, quality of anesthesia, post op analgesia after deflation of tourniquet, time of first analgesic drug and number of analgesic drug required in first 24 hrs. RESULT: Onset of effect was same in both groups. Intraoperative conditions and post operative analgesia were significantly better in Tramadol group. Consequently, number of doses of analgesic required in first 24 hours was less in Tramadol group. CONCLUSION: Addition of Tramadol has improved the quality of intravenous anaesthesia.
\end{abstract}

KEYWORDS: IVRA (Intra Venous Regional Anesthesia), Tramadol.

INTRODUCTION: Intravenous regional anesthesia (IVRA), a type of regional anesthesia was introduced by German surgeon August K.G. Bier (1908); thus the name, "Bier's Block". The main advantages of this technique are its simplicity, effectiveness, safety, reliability and being economical for day care and emergency surgery circumventing the problems of full stomach.

The ideal IVRA solution should have Rapid onset of sensory and motor block with prolonged analgesia after deflation of tourniquet. To achieve this, we evaluated effect of addition of an opioid, Tramadol08 to lignocaine in IVRA. Tramadol is a synthetic opioid analgesic with a unique dual mechanism of action. It exerts agonist properties at opiate receptors and also interferes with neurotransmitter reuptake ${ }^{02}$. Hence in this prospective, randomized controlled study we compared Tramadol (100mg) for its efficacy as an intra-operative, post-operative and preemptive analgesic when added to lignocaine $40 \mathrm{ml}$ solution in IVRA for upper arm orthopedic surgeries.

MATERIALS AND METHODS: A study of IVRA with Lignocaine compared with Lignocaine and Tramadol was carried out in 60 patients undergoing upper arm orthopedic surgery. 
Appropriate approval was taken from Hospital Ethics Committee. All patients selected were planned for forearm surgeries, of ASA Grade I or II, aged 15-65 years, Weighing 40-70 Kg. Patients were excluded from study when there was refusal for the procedure from patient's side, patients had hypersensitivity to local anesthetic agents, Opioids, NSAID drugs or when Patients were having past or present history of sickle cell disease, peripheral vascular disease, any neurological disease, hemolytic disease or any major systemic disease.

After detailed examination and informed consent, patients were randomly assigned in two groups of thirty patients each. Preservative free Lignocaine was used.

A. Lignocaine $0.5 \% 40 \mathrm{ml}$. (now onwards group L)

B. Lignocaine $0.5 \% 40 \mathrm{ml}+$ Tramadol $100 \mathrm{mg}$. (now onwards group LT)

A detailed history and thorough general and systemic examination and required investigations were done. Exanguination was done by using Esmarch Bandage after keeping limb elevated for 3- 5 minutes. IVRA was given using double tourniquet technique and using any of the one drug combination. First the upper tourniquet was inflated to $100 \mathrm{~mm}$ of $\mathrm{Hg}$ above the systolic blood pressure and drug was injected through angiocath on operative limb. After 10 mins, the lower cuff was inflated and the upper cuff was deflated. All patients were premedicated with inj. Midazolam $0.05 \mathrm{mg} / \mathrm{kg}$. IV. $10 \mathrm{~min}$. prior to inflation of tourniquet for anxiolysis. After completion of surgery, the tourniquet was deflated by intermittent deflation and reinflation method over a period of 2-3min. In no circumstances, tourniquet was released before 20 minutes after injection of drug. Hemodynamic monitoring was done continuously throughout procedure and stay in Post Anesthesia Care Unit. Thereafter, patients were followed every 4 hourly in ward. Any patient who had inadequate analgesia or lengthened procedure were converted into general anesthesia and excluded from study. Postoperatively for V.A.S. $\geq 5$, Diclofenac Sodium 3 cc. (75 mg.) IV was given as rescue analgesic. Postoperative nausea vomiting was treated with intravenous Ondansetron $4 \mathrm{mg}$ intravenously.

The following parameters were studied and observations noted.

1. Onset of sensory analgesia - The time taken for onset of loss of pain sensation by pin prick after injection of drug.

2. Onset of motor paralysis - Time from injection of drug to onset of motor paralysis $\leq$ grade II.

3. Intra operative condition -

a) Excellent -Indicated complete loss of touch, position sense, and pain sensation with no sensitivity to pin prick or deep pressure with marked or total paralysis of muscle.

b) Good - Loss of pain, touch and position sense but retained a sensory response to maximum pressure applied to the fingernail.

c) Moderate- anesthesia which was complete in most areas but incomplete in small region (patchy). Mild pain or discomfort during the reduction or during the operative procedure.

d) Poor/Failure- Failure to obtain anesthesia, moderate to marked pain when carrying out the manipulative or surgical procedure or both.

4. Total surgical time

5. Total Tourniquet Time, 
6. Time to first analgesic- Time between deflation of tourniquet and consumption of first analgesic dose (V.A.S. $\geq 5$ ).

7) Number of analgesics required- Total number of analgesic doses required in first 24 hours after deflation of tourniquet.

The results were statistically evaluated using SPSS and EPI Info software.

\section{RESULTS:}

\begin{tabular}{|l|l|l|}
\hline Group & Group L & Group LT \\
\hline Age in years Male & 37.5 & 37.8 \\
\hline Sex Female & $88 \%$ & $76 \%$ \\
\hline \multicolumn{1}{|c|}{$12 \%$} & $24 \%$ \\
\hline Weight in Kg. & 60.26 & 57.76 \\
\hline Duration of Surgery & $82.4 \pm 18.35$ & $79 \pm 6.99$ \\
\hline Duration of Tourniquet & $97.9 \pm 18.53$ & $94.33 \pm 7.5$ \\
\hline Onset of Analgesia(Sec) & $209.17 \pm 129$ & $62 \pm 18$ \\
\hline Onset of motor paralysis & $7.65 \pm 3.37$ & $6.86 \pm 2.56$ \\
\hline $\begin{array}{l}\text { Analgesia after deflation of } \\
\text { tourniquet }\end{array}$ & $10 \pm 4.6$ & $363 \pm 74.88$ \\
\hline Tourniquet discomfort & 06 & 01 \\
\hline Rash & 00 & 05 \\
\hline PONV & 00 & 03 \\
\hline Analgesics in 24 hrs & $3.7 \pm 0.59$ & $1.3 \pm 0.46$ \\
\hline
\end{tabular}

Demographically both the groups were comparable. There was no significant difference between duration of surgery and tourniquet time. Onset of sensory analgesia was significantly earlier in LT group ( $62 \pm 18$ secs compared to $209.17 \pm 129$ ) while onset of motor paralysis was similar in both groups. Incidence of tourniquet pain was significantly higher in group L (6) then group LT (1).

The post operative analgesia was significantly longer in group LT ( $10 \pm 4.6$ mins) then group L (363 $\pm 74.88 \mathrm{mins})$. Accordingly the number of analgesics consumed in post operative first 24 hours was significantly less in group LT $(1.3 \pm 0.46)$ then group L $(3.7 \pm 0.59)$. The incidence of rash (5) and PONV (3) was higher in group LT.

DISCUSSION: IVRA introduced by Sir August Bier (1908) and hence named BIER'S BLOCK, was made popular by Sir Holmes ${ }^{08}$. But it has fallen out of routine use due to the limitations of poor muscle relaxation, immediate post operative pain and risk of toxicity of local anesthetic agents. To improve post operative pain relief after IVRA, many adjuncts are tried. As the first family of analgesics, Opioids are a natural choice to try for the same. In this study, we have tried to study the effect of addition of an opioid, Tramadol to local anesthetic solution of IVRA on the parameters of onset of analgesia, quality of intraoperative analgesia, muscle relaxation, analgesia after deflation of tourniquet and number of analgesics required in first 24 hours.

The study was done in 60 of ASA Grade I/ II patients planned for forearm surgeries. They were randomly assigned in two groups of thirty patients each. 
A) Lignocaine $0.5 \% 40 \mathrm{ml}$. (group L) and B). Lignocaine $0.5 \% 40 \mathrm{ml}+$ Tramadol 100mg. (group LT)

Both the groups were comparable in age, sex \& weight.

This technique is less suitable for the lower limb due to the difficulty in venepuncture, especially in the affected limb, difficulty in retaining the tourniquet and higher dose of anesthetic agent required (Dawkins 1964, Mittal 1972) ${ }^{03,09}$. We selected all orthopedic patients undergoing surgeries in upper limb.

According to Holmes (1963), any suitable vein distal to the tourniquet can be used while Dawkins et al (1964) opines that site of injection should be as near the region of operation as possible ${ }^{03}$. Prithvi Raj (1972) proved by nerve conduction, radiopaque studies and radio isotope studies that distribution of contrast was similar whether cannula was inserted in to the median cubital vein at the elbow or at the dorsum of the hand ${ }^{10}$. We used veins on dorsum of hand and $22 \mathrm{G}$ angiocath in both groups. Anesthesia appeared from fingertip upward.

The choice of lignocaine as the local anesthetic agent and its dose has been suggested by many studies done before to be $3 \mathrm{mg} / \mathrm{kg}$ or $30-40 \mathrm{ml}$ of Lignocaine $0.5 \%$ solution ${ }^{07,08}$.In 1983, the American Food and Drug Administration (FDA) specifically contraindicated use of bupivacaine for IVRA following unfortunate incidents of cardiac toxicity followed by use of bupivacaine in IVRA.

We used $40 \mathrm{ml}$ of $0.5 \%$ lignocaine solution for both the groups. We used Esmarch bandage for all patients of both groups for exsanguinations.

Tourniquet pain is nociceptive, generated by activation of peripheral nociceptors and direct axonal stimulation of nervous trunks. We used double balloon tourniquet, applied on upper $2 / 3$ rd arm. The lower tourniquet was inflated after obtaining the analgesia below the upper tourniquet and then upper tourniquet deflated. The technique of using double balloon tourniquet is very effective in reducing pain due to tourniquet.

Onset of sensory analgesia was tested by loss of sensation to pin prick at least three separate areas innervated by 3 main nerves of upper extremity.

We observed mean time for onset of sensory analgesia for LT group to be $62 \pm 18$ seconds, While in group $\mathrm{L}$ it is significantly more at $209.17 \pm 129$ second $(\mathrm{P}<0.001)^{1}$.

The mean time for onset of muscle relaxation in group L was $7.65 \pm 3.37$ minutes while in Group LT $6.86 \pm 2.56$ minutes. There is no significant difference in onset of muscle relaxation in between two groups as is echoed by other studies $12,11,05$.

One of the major limiting factors has been the minimal or no residual post operative analgesia following IVRA $08,03,09$. Many adjuvants have been tried to counter this limitation. Kulkarni et al (1993) used Lignocaine+ Ketamine+ Gallamine04, Sunita Goel et al (2002) Lignocaine + Tramadol or Ketorolac ${ }^{06}$, Acalovschi et al Lignocaine + tramadol $^{1}$ and Reuben et al (1995) Lignocaine with ketorolac ${ }^{01}$. An opioid was considered to be a more acceptable choice because of its good analgesic efficiency. Tramadol is a synthetic opioid analgesic with a unique dual mechanism of action. It exerts agonist properties at opiate receptors and also interferes with neurotransmitter reuptake. We used Tramadol in dose of $100 \mathrm{mg}$ as adjuvant to local anesthetic solution.

POST OPERATIVE ANALGESIA: No significant post operative analgesia was found after deflation of tourniquet when only local anesthetic solution was used $08,09,03$. Various findings have been noted when different adjuvant were added to IVRA solution. Acolovschi et al (2001) demonstrated that the speed of recovery of sensory block was similar (pin prick and cold sensation) but for touch speed of recovery was delayed in Tramadol Lignocaine group ${ }^{1}$. He also 
showed that cold sensation recovered slower than the pin prick sensation. Sunita Goel et al (2002) also demonstrated that Tramadol assured significantly longer pain free interval of $16.8 \pm$ 9.07 hours $(\mathrm{p}<0.05)$ while Ketorolac had $12.9 \pm 8.48$ hours $^{06}$. We found analgesia after deflation of tourniquet significantly longer in Tramadol group (363 min.) than Lignocaine group (10 min.) $(\mathrm{P}<0.00001)$. Thus, it can be stated that addition of Tramadol to Lignocaine solution for IVRA definitely prolongs duration of analgesia after deflation of tourniquet. Another way to evaluate the post operative analgesia is total number of analgesics required in first 24 hours after the procedure. In our study the consumption of analgesic was significantly high in group L $3.7 \pm$ $0.59)$ than group LT $(1.3 \pm 0.46)$ in first 24 hours. $(\mathrm{P}<0.005)$. For group $\mathrm{L}$ the requirement of first and second analgesics was earlier (10 min.) than group LT (363 min.), showing tramadol has postoperative analgesic effect and also has a property of preemptive analgesia. Reuben et al (1995), Sunita Goel et al have also observed similar results.

ADVERSE EFFECTS: We did not find any adverse effect like central nervous system toxicity, restlessness, bradycardia, muscle fasciculation, pain or burning sensation at the site of injection. But we found, in 5 patients out of thirty in LT group, rash below level of tourniquet after injection of drug on the operating forearm. The rash disappeared within ten minutes after its appearance and required no active treatment. The incidence of rash over forearm in LT group was significantly high than L group which had no incidence. Similarly, 3 out of 30 suffered from PONV in LT group, but none required any treatment. The difference was not significant from $\mathrm{L}$ group where no one suffered from PONV. $(\mathrm{P}<0.05)$.

CONCLUSION: Addition of Tramadol $100 \mathrm{mg}$. with $0.5 \%$ Lignocaine for IVRA substantially augments the speed of sensory block, improves intraoperative analgesia and prolongs the duration of analgesia after deflation of tourniquet without any significant side effects.

\section{REFERENCES:}

1. Acalovschi I, Cristea T. Intravenous regional anesthesia with meperidine.

2. Anesth Analg 1995; 81: 540 -3.

3. C Rhoda Lee, Donna McTavish and Eugene M. Sorkin. Tramadol a preliminary review of its pharmacodynamics and pharmacokinetic properties, and therapeutic potential in acute and chronic pain states. Drugs 46 (2): 313-340, 1993.

4. Dawkins OS, Russel ES, Adams AK, Hooper RL, Okicikosa DA, Flemming. Intravenous regional anesthesia. Canadian anaesthetist society Jn; 1964; 11; 243.

5. DK Kulkarni, R Gopinath, Y Rajender, RS Manimala. Intravenous regional anesthesia with different drug combinations: A comparative study. Ind. J. Anaesth. (41), 114, 1993.

6. Elhakim M. Sadek RA. Addition of atracurium to lidocainefor intravenous regional anesthesia. Acta Anaesthesiol Scand 1994; 38:54-4.

7. Goel Sunita N, Daftary Swati R, Pantavaidya Shanti H. Intravenous regional anesthesia using tramadol hydrochloride and ketorolac: a double blind control study. Indian J Anaesth. 2002: 46 (5): 369-72.

8. Harris WH, Slater EM, Bell HM. Regional anesthesia by the intravenous route. JAMA 1965; 194: 1273-6.

9. Holmes CM. Intravenous regional analgesia a useful method of producing analgesia of the limbs. Lancet 1963; 1: 2457. 
10. Mittal NK, Kakkar SN. Intravenous regional anesthesia a clinical study.

a. Indian Journal of Anesthesia 1972; May; 185-92.

11. Raj PP, Garcia CE, Burleson JW, Jenkins MT. The site of action of intravenous regional anesthesia. Anesth Analg 1972; 51: 77686.

12. Reuben SS, Steinberg RB, Kreitzer JM, Duprat KM. Intravenous regional anesthesia using lidocaine and ketorolac. Anesth Analg 1995; 81: 110 -3.

13. Sukhani R, Garcia CJ, Munhall RJ, Winnie AP, Rodvold KA. Lidocaine disposition following intravenous regional anesthesia with different tourniquet deflation technique. Anesth Analg. 1989 May; 68(5): 633-7. 